\title{
Dose effect of oral Saccharomyces boulardii treatments on morbidity and mortality in immunosuppressed mice
}

\author{
L. A. PERET FILHO, F. J. PENNA, E. A. BAMBIRRA* and J. R. NICOLI† \\ Departamento de Pediatria and *Departamento de Anatomia Patológica, Faculdade de Medicina and \\ †Departamento de Microbiologia, Instituto de Ciências Biológicas, Universidade Federal de Minas Gerais, \\ Belo Horizonte MG, Brazil
}

\begin{abstract}
Survival, weight loss, translocation and histological alterations in the terminal ileum, liver and spleen were studied in mice simultaneously immunosuppressed with cyclophosphamide and treated or not with Saccharomyces boulardii until the death of all animals. The animals were divided into five groups: C1 (not immunosuppressed, not treated); C2 (immunosuppressed, not treated); B1 (immunosuppressed, treated with $S$. boulardii $10.0 \mathrm{mg}$ ); B2 (immunosuppressed, treated with $S$. boulardii $1.0 \mathrm{mg}$ ) and B3 (immunosuppressed, treated with $S$. boulardii $0.1 \mathrm{mg}$ ). Survival was higher in group B3 than in the other immunosuppressed groups. Weight loss was observed for all groups except C1. By day 7, some animals from each group were killed by ether inhalation for the determination of bacterial translocation and histopathological examination. Bacterial translocation to the liver was lower in groups $\mathrm{C} 1$ and $\mathrm{B3}$ than in the other groups. The highest translocation to the liver and spleen was observed in group B1. Low $S$. boulardii translocation was observed in some animals, principally to the mesenteric lymph nodes. Histopathological examination showed a decrease in epithelial cell turnover with villus length reduction and loss of brush borders in group C2. Relative protection against these alterations was obtained when the animals were treated with the yeast, independently of the dose. Higher expression of the lymphoid component was also noted in the ileal lamina propria, liver and spleen of mice treated with the yeast, together with activation of the reticulo-endothelial system, when compared with group C2 where lymphocyte depletion was observed. This study suggests a relative protection of immunosuppressed animals by treatment with $S$. boulardii, but this phenomenon was inversely proportional to the yeast dose.
\end{abstract}

\section{Introduction}

Aggressive cytostatic treatment applied for remission induction therapy in leukaemic patients results in increasing granulocytopenic episodes, as well as serious damage to the epidermal and mucosal barriers and deterioration of humoral defence and cell immunity. Therefore, severe bacterial and deep mycotic infections are the main cause of complications and death in patients with acute leukaemia [1]. The majority of these severe infections are of endogenous origin, i.e., caused by micro-organisms that have colonised the mucosal surface of the gastrointestinal (GI) tract and then have translocated.

Received 4 Feb. 1997; revised version received 18 June 1997; accepted 1 July 1997.

Corresponding author: Dr J. R. Nicoli.
Translocation is defined as the passage of viable microbes from the GI tract across the mucosal barrier to extra-intestinal sites [2]. Bacterial translocation, as measured by the appearance of viable bacteria in extra-intestinal organs, is promoted by three major mechanisms: (i) physical disruption of the mucosal barrier [3], such as occurs with ischaemia and reperfusion injury during endotoxic or haemorrhagic shock; (ii) intestinal bacterial overgrowth following disruption of the GI ecology by oral antibiotics, protein malnutrition or shock; and (iii) decreased host immune defences [4], resulting from immunosuppressive drugs or diseases such as cancer and AIDS.

The growing number of immunological incompetence situations promoting translocation has justified research on immunotherapeutic agents which may 
potentiate the resistance of an incompetent host. Biotherapeutic agents (probiotics) are known to offer an alternative to conventional antimicrobial agents to which many pathogenic micro-organisms eventually develop resistance [5]. A study in healthy human volunteers showed that oral administration of Saccharomyces boulardii activates both the complement and reticulo-endothelial systems [6]. No serious adverse effects have been reported with the biotherapeutic agents tested to date [5]. An obvious concern in the use of biotherapeutic agents is administration to severely immunocompromised or debilitated patients such that invasion into the systemic compartment might occur. However, there is limited information on the use of biotherapeutic agents in immunocompromised hosts. In mice immunosuppressed with prednisolone, orally administered $S$. boulardii decreased both the incidence of Candida albicans translocation to the mesenteric lymph nodes (MLN), liver and kidneys, and the number of translocating C. albicans per gram of MLN, spleen and kidneys [7]. $S$. boulardii was used in a double-blind, placebocontrolled trial in patients infected with HIV and presenting with chronic diarrhoea; $56 \%$ of the patients treated with the yeast had resolution of this diarrhoea compared with $6 \%$ of the patients on placebo [8]. Beyond the initial clinical trials of effectiveness against various diseases, biotherapeutic agents need to be characterised with the same dose-ranging studies expected for standard chemical investigational drugs. The published studies have rarely presented data on dose-dependent efficacy of the agents.

This study examined the effect of different oral doses of $S$. boulardii on survival, microbial translocation and histological alterations in the terminal ileum, liver and spleen of mice immunosuppressed with cyclophosphamide.

\section{Materials and methods}

\section{Mice}

Male NMRI Swiss mice (Central Institute for Animal Breeding, Hannover, Germany), 1 month old, were used (initial weight $20.04 \mathrm{SD} 1.36 \mathrm{~g}$ ). The animals were given a commercial diet (Nuvital, Curitiba PR, Brazil) for rodents and water ad libitum.

\section{Experimental design}

The animals were divided into five groups: $\mathrm{Cl}$ - not immunosuppressed, not treated; $\mathrm{C} 2$ - immunosuppressed, not treated; B1 - immunosuppressed, treated with $10.0 \mathrm{mg}$ of $S$. boulardii; B2 - immunosuppressed, treated with $1.0 \mathrm{mg}$ of $S$. boulardii; and B3 immunosuppressed, treated with $0.1 \mathrm{mg}$ of $S$. boulardii. The mice were weighed at the beginning of the experiment and at day 7 , when some of the animals from each group were killed by ether inhalation to determine translocation and for histopathological examination. The cumulative mortality was observed in the remaining mice until the end of the experiment, at day 30 .

\section{Treatments}

In the immunosuppressed model, mice were given cyclophosphamide (Enduxan; Shionogi and Co. Ltd, Osaka, Japan) $100 \mathrm{mg} / \mathrm{kg}$ by intraperitoneal injection on alternate days. When mice were given $S$. boulardii (B groups), the treatment was given concurrently by daily intragastric intubation with $0.1 \mathrm{ml}$ of yeast suspension during the same period. Lyophilised $S$. boulardii (Floratil; Merck SA, Rio de Janeiro RJ, Brazil) were suspended to the appropriate concentration in sterile PBS. In control animals (C groups), the same schedule was followed with saline for injection or intubation, or both.

\section{Microbial translocation test}

The MLN, liver and spleen of each mouse killed after 7 days were removed aseptically, weighed and homogenised in a sterile grinding mortar. Ten-fold dilutions were performed and used for bacterial and yeast counts by plating on to Brain Heart Infusion Agar (Difco) and Sabouraud Dextrose Agar (Difco), respectively. Plates were incubated overnight or for $72 \mathrm{~h}$ at $37^{\circ} \mathrm{C}$ before bacterial and yeast counts, respectively. Bacterial translocation was considered to be positive for values $\leqslant 10^{2} \mathrm{cfu} / \mathrm{g}$ of tissue for liver and spleen and $10^{3} \mathrm{cfu} / \mathrm{g}$ of tissue for MLN. For $S$. boulardii translocation, the presence of any number of colonies was considered positive.

\section{Histopathological examination}

Fragments of the terminal ileum, liver and spleen were fixed with formaldehyde $4 \%$ and processed for paraffin embedding, and histopathological sections $(3-5 \mu \mathrm{m})$ were stained with haematoxylin-eosin. The slides were all examined by the same pathologist, who was unaware of the experimental conditions of each group. The slides were coded and decoded only after the report had been written.

\section{Statistical analysis}

The Kaplan Meier method and Wilcoxon and log-rank tests were used to compare survival times. Differences in translocation proportions were assessed by the Fisher's exact test. Comparisons of weight gain or loss were made by analysis of variance and co-variance and the Duncan multiple comparison method. All statistical analyses were performed with the Statistical Analysis System software (SAS Institute, Cary, NC, USA). 


\section{Results}

\section{Survival}

Fig. 1 shows the life-table survival curves (KaplanMeier method) for the different experimental groups and the $\mathrm{C} 2$ control group. Survival was significantly higher $(\mathrm{p}=0.0255)$ in group B3 $(0.1 \mathrm{mg}$ of $S$. boulardii) when compared with the other immunosuppressed groups (Wilcoxon test). There was no mortality in the $\mathrm{C} 1$ control group.

\section{Weight variation}

During the experiment, a weight loss was observed in all immunosuppressed groups $(-4.03,-2.39,-3.40$, and $-3.81 \mathrm{~g}$ for $\mathrm{C} 2, \mathrm{~B} 1, \mathrm{~B} 2$ and $\mathrm{B} 3$, respectively) and a weight gain in the $\mathrm{Cl}$ control group $(+2.79 \mathrm{~g})$.

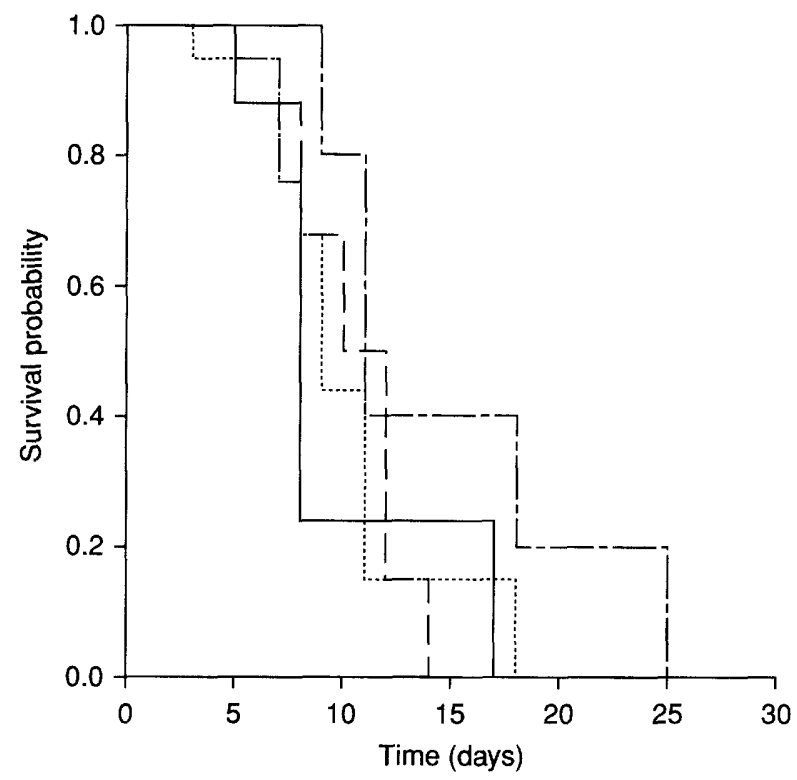

Fig. 1. Life-table survival curves (Kaplan-Meier method) for immunosuppressed, untreated mice ( 2 2, - $)$, immunosuppressed mice treated with $10.0 \mathrm{mg}$ of $S$. boulardii (B1, - - ), immunosuppressed mice treated with $1.0 \mathrm{mg}$ of $S$. boulardii (B2, - - - ) and immunosuppressed mice treated with $0.1 \mathrm{mg}$ of $S$. boulardii (B3, - - - ).
Despite the progressively higher weight loss with a decreased yeast dose, there was no significant difference between these values for the immunosuppressed groups. Analysis of co-variance with weighted least squares showed a statistically significant difference only between the two control groups $\mathrm{C} 1$ and $\mathrm{C} 2$ $(\mathrm{p}<0.05)$.

\section{Translocation}

Table 1 shows the bacterial translocation index to MLN, liver and spleen for each group tested after treatment for 7 days. Statistical comparison was performed by the Fisher's exact test between individual data of each group and for the same organ. The bacterial translocation index to the MLN was statistically similar for all groups. Translocation to the liver was lower in groups $\mathrm{C} 1$ and $\mathrm{B} 3$ when compared to the other groups $(p=0.0021)$. The greatest translocation to the liver was observed in group B1 $(10.0 \mathrm{mg}$ of $S$. boulardii). Higher bacterial translocation was also observed in the spleen of group B1 animals, but in this case the results with the $\mathrm{C} 2, \mathrm{~B} 2$ and $\mathrm{B} 3$ groups were statistically similar $(p=0.00015)$. When statistically similar data were grouped $(\mathrm{C} 1-\mathrm{B} 3 ; \mathrm{C} 2-\mathrm{B} 2 ; \mathrm{B} 1$ for liver and $\mathrm{C} 1 ; \mathrm{C} 2-\mathrm{B} 2-\mathrm{B} 3$; $\mathrm{B} 1$ for spleen) and these groups were compared again by Fisher's exact test, the results obtained above for individual animals were confirmed and reinforced $(p=0.000381$ for grouped liver data and $p=0.000011$ for grouped spleen data). Translocation of $S$. boulardii was observed principally to the MLN. Translocation of the yeast to liver and spleen occurred rarely and always in animals with simultaneous significant bacterial translocation. In this case, the translocation levels of $S$. boulardii never exceeded 2.0 or $1.0 \log _{10} \mathrm{cfu} / \mathrm{g}$ of organ for MLN or liver and spleen, respectively. There was no translocation of yeasts of the normal GI microbiota in the $\mathrm{C} 2$ group (data not shown).

\section{Histopathology}

Histopathological examination of the terminal ileal mucosa showed a decrease in epithelial cell turnover, flattened enterocytes and brush border discontinuity in

Table 1. Bacterial translocation index from the GI tract to mesenteric lymph nodes (MLN), liver and spleen

\begin{tabular}{|c|c|c|c|c|}
\hline \multirow[b]{2}{*}{ Groups } & \multicolumn{3}{|c|}{ Number $(\%)$ of positive organs } & \multirow[b]{2}{*}{ Number of mice tested } \\
\hline & MLN & liver & spleen & \\
\hline $\mathrm{Cl}$ & $2(14.3)^{*}$ & $3(21.4)^{*}$ & $0(0.0)^{*}$ & 14 \\
\hline $\mathrm{C} 2$ & $5(22.7)^{*}$ & $14(63.6)^{\dagger}$ & $12(54.6)^{\dagger}$ & 22 \\
\hline B1 & $6(50.0)^{*}$ & $11(91.7)^{\ddagger}$ & $10(83.3)^{\ddagger}$ & 12 \\
\hline B2 & $2(16.7)^{*}$ & $6(50.0)^{\dagger}$ & $6(50.0)^{\dagger}$ & 12 \\
\hline B3 & $3(20.0)^{*}$ & $5(33.3)^{*}$ & $6(40.0)^{\dagger}$ & 15 \\
\hline $\mathrm{p}$ & 0.289 & 0.0021 & 0.00015 & \\
\hline
\end{tabular}

$\mathrm{C} 1$, non-immunosuppressed, non-treated mice; $\mathrm{C} 2$, immunosuppressed, untreated mice; B1, immunosuppressed mice treated with 10.0 mg of $S$. boulardii; B2, immunosuppressed mice treated with $1.0 \mathrm{mg}$ of $S$. boulardii; and B3, immunosuppressed mice treated with $0.1 \mathrm{mg}$ of $S$. boulardii

${ }^{* \dagger}$ Significantly different, $\mathrm{p}<0.05$.

p-value, comparison of individual data for the same organ by the Fisher's exact test. 

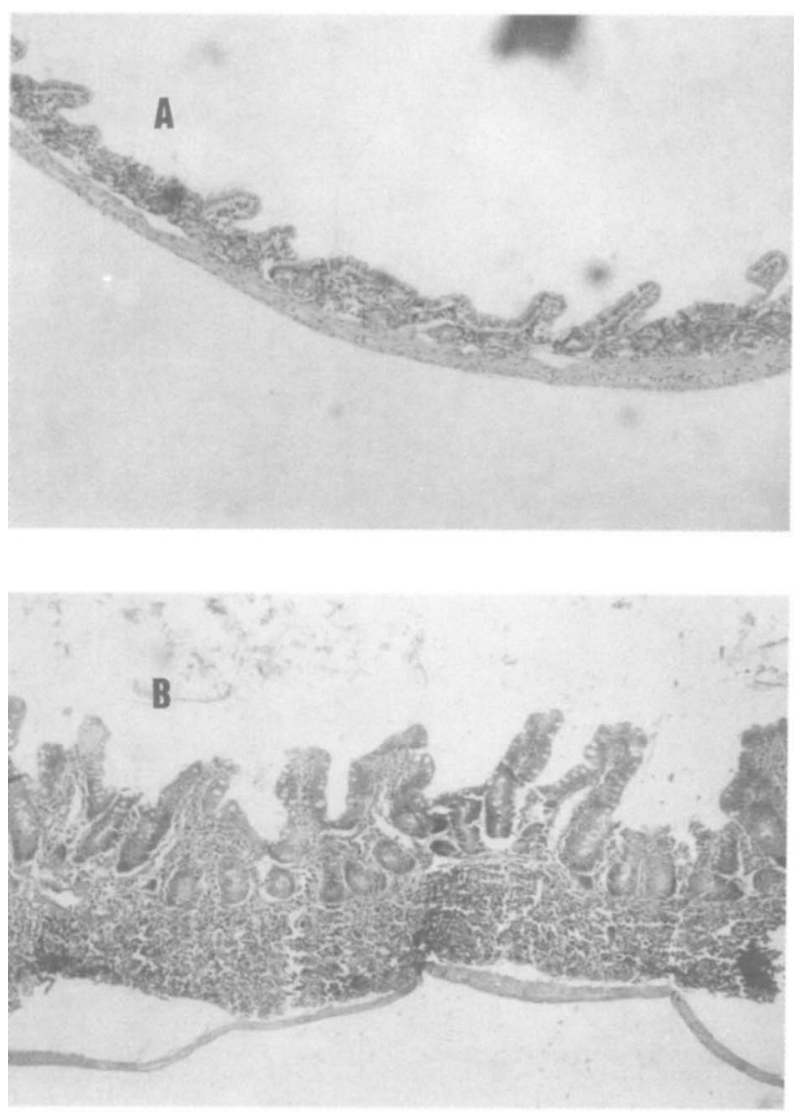

Fig. 2. Histopathological aspect of terminal ileal mucosa from: A, immunosuppressed, untreated mice (C2); B, immunosuppressed mice treated with $0.1 \mathrm{mg}$ of $S$. boulardii (B3). (Haematoxylin and eosin, $\times 63$ ).

group C2 (Fig. 2A). Relative protection against these alterations was obtained when the animals were treated with the yeast, independently of the dose (Fig. 2B). Higher expression of the lymphoid component in the ileal lamina propria, liver and spleen was also noted in mice treated with the yeast, including an activation of the reticulo-endothelial system, when compared with group $\mathrm{C} 2$ where lymphocyte depletion was observed (Fig. 2A and B, Fig. 3A and B).

\section{Discussion}

The GI tract has been demonstrated to be a reservoir for bacteria causing life-threatening infections in cancer patients undergoing chemotherapy [9], in bone marrow recipients [10] and in patients with AIDS [11]. Bacterial translocation from the GI tract is an important early step in the pathogenesis of opportunist infections for these debilitated patients.

It seems that all the components of the host immune system - including mucosal, cell-mediated and humoral immunities - are involved in reducing bacterial translocation. Secretory immunoglobulin A ( $\operatorname{sg} \mathrm{A})$ is thought to inhibit the close association of bacteria with the mucosal epithelium, and so to reduce
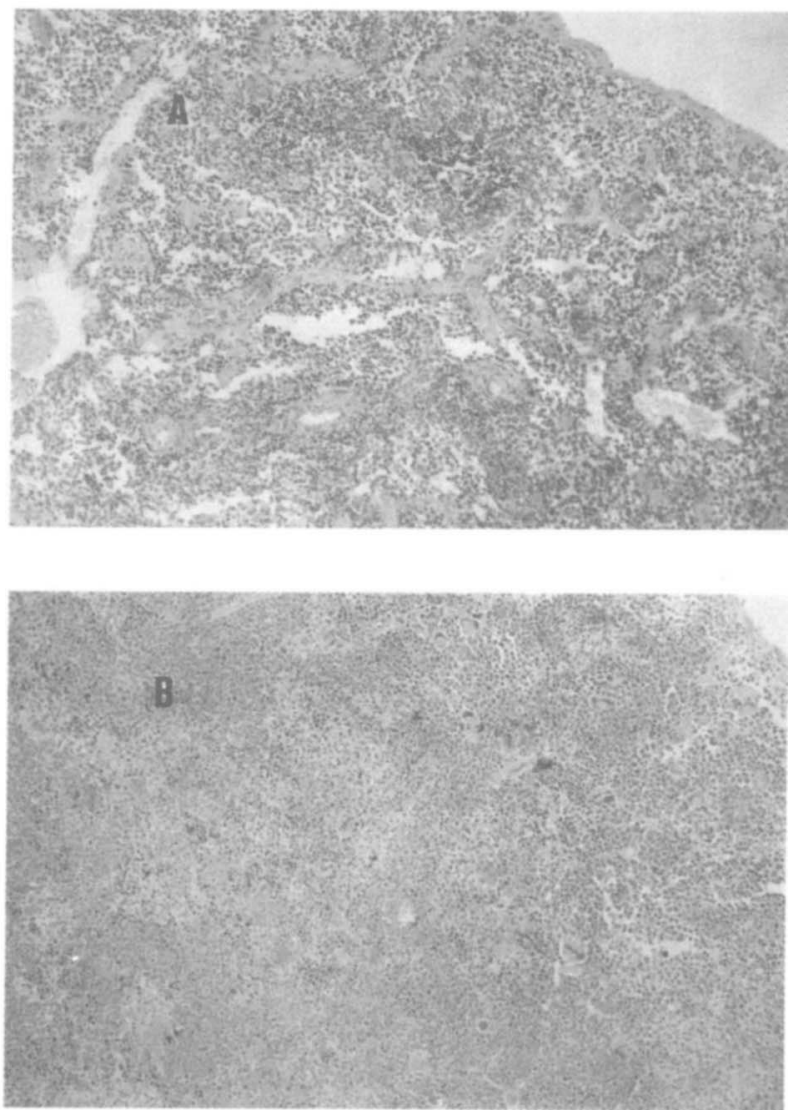

Fig. 3. Histopathological aspect of spleen parenchyma from: A, immunosuppressed, untreated mice (C2); B, immunosuppressed mice treated with $0.1 \mathrm{mg}$ of $S$. boulardii (B3). (Haematoxylin and eosin, $\times 160$ ).

bacterial penetration. Cell-mediated immunity in the lamina propria and in lymphoid organs provides a second line of host defence against bacterial translocation. The complete depletion of either $\mathrm{CD}^{+}$or $\mathrm{CD}^{+} \mathrm{T}$ cells, or both, in the intestinal epithelium, lamina propria and MLN promoted bacterial translocation [12]. Serum immunoglobulins probably act as opsonins to increase the effectiveness of phagocytosis by macrophages and polymorphonuclear leucocytes and subsequent killing of translocating bacteria. In the case of a successful translocation, the reticuloendothelial system (RES) plays a key role in removing micro-organisms from the circulation [13], and hepatic phagocytosis by Kupffer cells accounts for $>80 \%$ of RES function [14].

In this era of AIDS and selective immunosuppressive therapies which promote translocation, there is substantial interest in developing new methods of immune stimulation such as the use of probiotics. However, before a new substance can be used as an immunomodulator, it will have to be checked, not only as regards its efficiency in the enhancement of the immune response, but also as regards the absence of adverse side-effects for the host.

The results of the present study suggest that at a lower 
dose $(0.1 \mathrm{mg}) \quad S$. boulardii can reduce bacterial translocation from the GI tract (Table 1) and consequently the mortality (Fig. 1) of mice immunosuppressed with cyclophosphamide. It is not known precisely how oral $S$. boulardii treatment protects the host against infections by either their indigenous GI microbiota or by recently acquired exogenous microbes, although several properties of the yeast may contribute to its efficacy. For example, $S$. boulardii is reported to antagonise populations of certain microbial pathogens such as C. albicans [15], but not of Salmonella typhimurium or Shigella flexneri [16]. On the other hand, another important effect of the yeast treatment is the stimulation of non-specific antiinfectious defences such as the complement system and the phagocytic mononuclear system [6]. Glucan and other Saccharomyces extracts are important RES stimulators. The effects of these yeast products have been partially clarified. Proliferation of pluripotent haematopoietic stem cells, granulocyte-macrophage colony-forming cells and erythroid colony-forming cells in murine bone marrow has been demonstrated after glucan administration [17-19]. Furthermore, after glucan administration, there is an increased production of interleukin 1 either by a direct effect on macrophages or indirectly by an increase in colony stimulating factor(s) production from glucan-stimulated T-cells [20]. The protective effect obtained with oral $S$. boulardii treatment and the simultaneous activation of the reticulo-endothelial system was confirmed histopathologically. The higher expression of lymphoid components in the ileal lamina propria, liver and spleen of mice treated with the yeast was also observed in previous works [16, 21]. A significant increase in Escherichia coli blood clearance, as an indicator of the host's mononuclear phagocyte system activity, was found in gnotoxenic mice mono-associated with $S$. boulardii when compared with germfree animals [22].

In group $\mathrm{Cl}$ mice, a low 'physiological' bacterial translocation was noted similar to the rates reported by others [2]. As suggested by Berg [23], this very low level of translocation of indigenous bacteria could be beneficial in priming the host immune response to improve defence against overt or opportunist pathogens.

Consequently, although the results presented here are only preliminary and the experiments were conducted on an animal model, the reduction of bacterial translocation and mortality by a low oral dose of $S$. boulardii suggests a possible therapeutic benefit of the probiotic for patients at risk for opportunist infections by their indigenous GI microbiota.

This work was supported by grants from Merck SA (Dr A. G. Fernandes, Rio de Janeiro RJ, Brazil), Fundaçäo de Amparo à Pesquisa do Estado de Minas Gerais (FAPEMIG), Financiadora de Estudos e Projetos (FINEP) and Conselho Nacional de Desenvolvimento Tecnológico e Científico (CNPq). The authors are grateful to
Maria Gorete Barbosa Ribas, Antônio Carlos Prudente do Pinho Jr, Cinthia Faiçal Parenti, Elaine Souza Barros and Luciana Diniz Silva for valuable technical assistance.

\section{References}

1. Singer C, Kaplan MH, Armstrong D. Bacteremia and fungemia complicating neoplastic disease. A study of 364 cases. $\mathrm{Am} \mathrm{J}$ Med 1977; 62: 731-742.

2. Berg RD, Garlington AW. Translocation of certain indigenous bacteria from the gastrointestinal tract to the mesenteric lymph nodes and other organs in a gnotobiotic mouse model. Infect Immun 1979; 23: 403-411.

3. Morehouse JL, Specian RD, Stewart JJ, Berg RD. Translocation of indigenous bacteria from the gastrointestinal tract of mice after oral ricinoleic acid treatment. Gastroenterology 1986; 91 : 673-682.

4. Berg RD, Wommack E, Deitch EA. Immunosuppression and intestinal bacterial overgrowth synergistically promote bacterial translocation. Arch Surg 1988; 123: 1359-1364.

5. Elmer GW, Surawicz CM, McFarland LV. Biotherapeutic agents: a neglected modality for the treatment and prevention of selected intestinal and vaginal infections. JAMA 1996; 275 : 870-876.

6. Caetano JAM, Parames MT, Babo MJ et al. Immunopharmacological effects of Saccharomyces boulardii in healthy human volunteers. Int J Immunopharmacol 1986; 8: 245-259.

7. Berg R, Bernasconi P, Fowler D, Gautreaux M. Inhibition of Candida albicans translocation from the gastrointestinal tract of mice by oral administration of Saccharomyces boulardii. $J$ Infect Dis 1993; 168: 1314-1318.

8. Saint-Marc T, Rossello-Prats L, Touraine JL. Efficacité de Saccharomyces boulardii dans le traitement des diarrhées du SIDA. Ann Med Interne (Paris) 1991; 142: 64-65.

9. Cesario TL, Slater LM, Armentrout SA, Thrupp LD, Tilles JG. Septicemia in acute leukemia. Med Pediatr Oncol 1978; 5: 193-203.

10. Klatersky J. Therapy of bacterial infections in cancer patients. In: Verhoeft P, Peterson PK, Quie PG (eds) Infections in the immunocompromised host - Pathogenesis, prevention and therapy. Amsterdam, Elsevier/North Holland Publishing Co. 1980: 207-299.

11. Witt DJ, Craven DE, McCabe WR. Bacterial infections in adult patients with the acquired immune deficiency syndrome (AIDS) and AIDS-related complex. Am J Med 1987; 82: 900-906.

12. Gautreaux MD, Deitch EA, Berg RD. T lymphocytes in host defense against bacterial translocation from the gastrointestinal tract. Infect Immun 1994; 62: 2874-2884.

13. Katz S, Jimenez MA, Lehmkuhler WE, Grosfeld JL. Liver bacterial clearance following hepatic artery ligation and portacaval shunt. J Surg Res 1991; 51: 267-270.

14. Saba TM. Physiology and physiopathology of the reticuloendothelial system. Arch Intern Med 1970; 126: 1031-1052.

15. Ducluzeau R, Bensaada M. Effet comparé de l'administration unique ou en continu de Saccharomyces boulardii sur l'établissement de diverses souches de Candida dans le tractus digestif de souris gnotoxéniques. [Comparative effect of a single or continuous administration of "Saccharomyces boulardii" on the establishment of various strains of "Candida" in the digestive tract of gnotobiotic mice.] Ann Microbiol 1982; 133: 491-501.

16. Rodrigues ACP, Nardi RM, Bambirra EA, Vieira EC, Nicoli JR. Effect of Saccharomyces boulardii against experimental oral infection with Salmonella typhimurium and Shigella flexneri in conventional and gnotobiotic mice. $J$ Appl Bacteriol 1996; 81: 251-256.

17. Matsuo T, Kurahashi $Y$, Nishida $S$, Onodera $C$, Izawa $M$, Hamuro J. Effect of lentinan, a specific T-cell adjuvant, on murine granulopoiesis and its roles on antitumor effect. Int $J$ Immunopharmacol 1982; 4: 269 (Abstract).

18. Patchen M, MacVittie T. Effects of a soluble glucan on hemopoietic stem cell proliferation. Int $J$ Immunopharmacol 1982; 4: 273 (Abstract)

19. Way CF, Dougherty WJ, Cook J. Inhibition of glucan induced hepatic granuloma formation by indomethacin or essential fatty acid deficiency (EFAD). Int J Immunopharmacol 1982; 4: 269 (Abstract) 
20. Hamuro J, Akiyama Y, Iguchi Y, Isawa M, Matsuo T. Distinct roles of serum factors induced by a $T$ cell specific immune adjuvant lentinan in cellular immune responses. Int $J$ Immunopharmacol 1982; 4: 268 (Abstract).

21. Dias RS, Bambirra EA, Silva ME, Nicoli JR. Protective effect of Saccharomyces boulardii against the cholera toxin in rats. Braz J Med Biol Res 1995; 28: 323-325.
22. Neumann E, Oliveira MA, Péret LA et al. The probiotic stimulation of the host mononuclear phagocyte system in gnotobiotic and conventional mice. XIIth International Symposium on Gnotobiology 1996, Honolulu, Hawaii: Abstract S-28.

23. Berg $\mathrm{RD}$. Bacterial translocation from the gastrointestinal tract. Trends Microbiol 1995; 3: 149-154. 